\title{
SECRECY CAPACITY OF MISO GAUSSIAN WIRETAP CHANNEL WITH A COOPERATIVE JAMMER
}

\author{
S. Ali. A. Fakoorian and A. Lee Swindlehurst \\ Center for Pervasive Communications and Computing, UCI \\ afakoori, swindle@uci.edu
}

\begin{abstract}
We study the Gaussian MISO wiretap channel with a transmitter, a legitimate receiver, an eavesdropper and an external helper, where the transmitter and the helper have an arbitrary number of antennas but the intended receiver and the eavesdropper have only one antenna. The transmitter sends confidential messages to its intended receiver, while the helper transmits jamming signals independent of the source message to confuse the eavesdropper. The jamming signal is assumed to be treated as noise at both the intended receiver and the eavesdropper. Assuming Gaussian signaling at the helper, we obtain the optimal beamformers at the transmitter and the helper that achieve the secrecy capacity.
\end{abstract}

\section{INTRODUCTION}

Recent information-theoretic research on secure communication has focused on enhancing security at the physical layer. The wiretap channel, first introduced and studied by Wyner [1], is the most basic physical layer model that captures the problem of communication security. This work led to the development of the notion of perfect secrecy capacity, which quantifies the maximum rate at which a transmitter can reliably send a secret message to its intended recipient, without it being decoded by an eavesdropper. The secrecy capacity of a Gaussian wiretap channel, in which the outputs of the legitimate receiver and the eavesdropper are corrupted by additive white Gaussian noise, has been addressed and solved for in [2]-[5].

It was shown in [6] that, for a wiretap channel without feedback, a non-zero secrecy capacity can only be obtained if the eavesdropper's channel is of lower quality than that of the intended recipient. Otherwise, it is infeasible to establish a secure link under Wyner's wiretap channel model. In such situations, one approach is to exploit user cooperation in facilitating the transmission of confidential messages from the source to the destination. In [7]-[12], for example, a four-terminal relay-eavesdropper channel is considered, where a source wishes to send messages to a destination while

This work was supported by the U.S. Army Research Office under the Multi-University Research Initiative (MURI) grant W911NF-07-1-0318. leveraging the help of a relay/helper node to hide the messages from the eavesdropper. While the relay can assist in the transmission of confidential messages, its computational cost may be prohibitive and there are difficulties associated with the coding and decoding schemes at both the relay and the intended receiver. Alternatively, a cooperating node can be used as a helper that simply transmits jamming signals, independent of the source message, to confuse the eavesdropper and increase the range of channel conditions under which secure communications can take place. The strategy of using a helper to improve the secrecy of the source-destination communication is generally known as cooperative jamming $[7,8]$ or noise-forwarding [9] in prior work.

In [7], the scenario where multiple single-antenna users communicate with a common receiver (i.e., the multiple access channel) in the presence of an eavesdropper is considered, and the optimal transmit power allocation that achieves the maximum secrecy sum-rate ia obtained. The work of [7] shows that any user prevented from transmitting based on the obtained power allocation can help increase the secrecy rate for other users by transmitting artificial noise to the eavesdropper (cooperative jamming). In [8], a sourcedestination system in the presence of multiple helpers and multiple eavesdroppers is considered, where the helpers can transmit weighted jamming signals to degrade the eavesdropper's ability to decode the source. While the objective is to select the weights so as to maximize the secrecy rate under a total power constraint, or to minimize the total power under a secrecy rate constraint, the results in [8] yield sub-optimal weights for both single and multiple eavesdroppers, due to the assumption that the jamming signal must be nulled at the destination. The noise forwarding scheme of [9] requires that the interferer's codewords be decoded by the intended receiver. A generalization of [7,8] and [9] is proposed in [10], in which the helper's codewords do not have to be decoded by the receiver. While all the prior work in [7]-[10] assumes single antenna nodes (SISO model), [11] assumes the case of multiple antennas at both the transmitter and the helper (MISO case), and proposes an iterative algorithm that jointly optimizes the transmitter and helper beamformers. The MIMO case with multiple cooperative jammers was studied in [12], in which the jammers aligned their interference to lie within a 
pre-specified "jamming subspace" at the receiver, but the dimensions of the subspace and the power allocation were not optimized.

In this paper, we also address the MISO case, where the transmitter and helper are equipped with multiple antennas. The transmitter sends confidential messages to its intended receiver, while the helper node assists the transmitter by sending jamming signals independent of the source message to confuse the eavesdropper. The jamming signal from the helper is not decoded by the intended receiver and is treated as noise at both the intended receiver and the eavesdropper. In this work, we obtain the optimal beamforming vectors of the transmitter and the eavesdropper that achieve the secrecy capacity.

The remainder of the paper is organized as follows. In Section 2, we describe the system model for the helper-assisted Gaussian MISO wiretap channel and formulate the problem to be solved. In Section 3, we derive the optimal beamformers for the transmitter and the helper that achieve the secrecy capacity. Numerical results in Section 4 are presented to illustrate the proposed solution. Finally, Section 5 concludes the paper.

Notation: Throughout the paper, we use boldface lowercase letters to denote column vectors. Vector-valued random variables are written with non-boldface uppercase letters (e.g., $X$ ), while the corresponding non-boldface lowercase letter $(\mathbf{x})$ denotes a specific realization of the random variable. Scalar variables are written with non-boldface (lowercase or uppercase) letters. We use $(.)^{H}$ to represent the Hermitian (i.e., conjugate) transpose, and I the identity matrix. Mutual information between the random variables $A$ and $B$ is denoted by $I(A ; B)$, and $\mathcal{C N}\left(0, \sigma^{2}\right)$ represents the complex circularly symmetric Gaussian distribution with zero mean and variance $\sigma^{2}$.

\section{SYSTEM MODEL}

We consider a MISO wiretap channel that includes a transmitter, an intended receiver, a helping interferer and an eavesdropper. We use $n_{t}$ and $n_{h}$ to denote the number of antennas at the transmitter and helper, respectively; the intended receiver and eavesdropper each have a single antenna. The transmitter sends a confidential message $M$ to the intended receiver with the aid of the helper, in the presence of an eavesdropper. We assume that the helper does not know the confidential message and transmits only a Gaussian jamming signal which is not known at the intended receiver nor the eavesdropper and which is treated as noise at both receivers. The mathematical model for this scenario is given by:

$$
\begin{aligned}
& y_{1}=\mathbf{h}_{1}^{H} \mathbf{x}_{1}+\mathbf{g}_{2}^{H} \mathbf{x}_{2}+z_{1} \\
& y_{2}=\mathbf{h}_{2}^{H} \mathbf{x}_{2}+\mathbf{g}_{1}^{H} \mathbf{x}_{1}+z_{2},
\end{aligned}
$$

where $\mathbf{x}_{1} \in \mathbb{C}^{n_{t} \times 1}$ is an information signal, $\mathbf{x}_{2} \in \mathbb{C}^{n_{h} \times 1}$ is a jamming signal transmitted by the helper, and $z_{1} \sim \mathcal{C N}\left(0, \sigma_{r}^{2}\right)$, $z_{2} \sim \mathcal{C N}\left(0, \sigma_{e}^{2}\right)$ are Gaussian noise signals at the intended receiver and the eavesdropper, respectively. The column vectors $\mathbf{h}_{1}, \mathbf{g}_{1}$ represent complex channel gains from the transmitter to the intended receiver and eavesdropper, respectively, and $\mathbf{h}_{2}, \mathbf{g}_{2}$ represent complex channel gains from the helper to the eavesdropper and intended receiver, respectively. All channel gains are fixed throughout the entire transmission period, and are known to all the terminals.

The jamming signal is constrained by

$$
\operatorname{Tr}\left(E\left\{X_{2} X_{2}^{H}\right\}\right)=\operatorname{Tr}\left(\mathbf{K}_{w}\right) \leq P_{h}
$$

where $\mathbf{K}_{w} \succeq \mathbf{0}$ is the helper covariance matrix. The information signal power is constrained by

$$
\operatorname{Tr}\left(E\left\{X_{1} X_{1}^{H}\right\}\right)=\operatorname{Tr}\left(\mathbf{K}_{x}\right) \leq P_{t}
$$

where $\mathbf{K}_{x} \succeq \mathbf{0}$ is the input covariance matrix.

For the perfect secrecy constraint, the confidentiality of the message $M$ at the eavesdropper is guaranteed if [4]

$$
\lim _{N \rightarrow \infty} \frac{1}{N} I\left(M \mid y_{2}^{N}\right)=0
$$

where $N$ is the number of channel uses to send message $M$. As mentioned before, we assume Gauusian signaling for the helper. Thus the effective noise at both receivers is Gaussian and consequently the above MISO wiretap channel model is Gaussian. For this case, a Gaussian input signal is an optimal choice [4], and the secrecy capacity is given by

$$
C_{\text {sec }}=\max _{\mathbf{K}_{x} \succeq 0, \mathbf{K}_{w} \succeq 0}\left[I\left(X_{1} ; y_{1}\right)-I\left(X_{1} ; y_{2}\right)\right] .
$$

A matrix characterization of this optimization problem is given by:

$$
\begin{aligned}
C_{\text {sec }}=\max _{\mathbf{K}_{x} \succeq 0, \mathbf{K}_{w} \succeq 0} & \log \left(1+\frac{\mathbf{h}_{1}^{H} \mathbf{K}_{x} \mathbf{h}_{1}}{\sigma_{r}^{2}+\mathbf{g}_{2}^{H} \mathbf{K}_{w} \mathbf{g}_{2}}\right) \\
& -\log \left(1+\frac{\mathbf{g}_{1}^{H} \mathbf{K}_{x} \mathbf{g}_{1}}{\sigma_{e}^{2}+\mathbf{h}_{2}^{H} \mathbf{K}_{w} \mathbf{h}_{2}}\right)
\end{aligned}
$$

where the non-convex maximization problem is carried out under the power constraints given in (3) and (4).

Lemma 1: For a given $\mathbf{K}_{w}$, the maximum of (7) is given by

$$
C_{s e c}\left(\mathbf{K}_{w}\right)=\log \lambda_{t}
$$

where $\lambda_{t}$ denotes the largest generalized eigenvalue of the pencil

$$
\left(\mathbf{I}+\frac{P_{t} \mathbf{h}_{1} \mathbf{h}_{1}^{H}}{\sigma_{r}^{2}+\mathbf{g}_{2}^{H} \mathbf{K}_{w} \mathbf{g}_{2}}, \mathbf{I}+\frac{P_{t} \mathbf{g}_{1} \mathbf{g}_{1}^{H}}{\sigma_{e}^{2}+\mathbf{h}_{2}^{H} \mathbf{K}_{w} \mathbf{h}_{2}}\right) .
$$

Proof: For a given $\mathbf{K}_{w}$, the channel is equivalent to a simple MISO Gaussian wiretap channel, where the noise variances at the receiver and the eavesdropper are $\left(\sigma_{r}^{2}+\mathbf{g}_{2}^{H} \mathbf{K}_{w} \mathbf{g}_{2}\right)$ and 
$\left(\sigma_{e}^{2}+\mathbf{h}_{2}^{H} \mathbf{K}_{w} \mathbf{h}_{2}\right)$, respectively. The above lemma is a natural extension of [2] and [13, Theorem 3] for the standard MISO Gaussian wiretap channel.

It is easy to verify that $\lambda_{t} \geq 1$, as shown in [13, App. A]. Moreover, Lemma 1 states that for a given $\mathbf{K}_{w}$, the optimal $\mathbf{K}_{x}$ that achieves (8) is a rank-one, and hence Gaussian signaling in the form of beamforming is optimal [2]. More precisely, the transmit signal can be represented as $\mathbf{x}_{1}=\mathbf{w}_{t} s$ where $s$ represents a Gaussian encoded information symbol broadcast by the transmitter using the beamforming vector $\mathbf{w}_{t}$, and $\mathbf{w}_{t}$ is a normalized eigenvector of the pencil (9) corresponding to $\lambda_{t}$ and scaled so that $\mathbf{w}_{t}^{H} \mathbf{w}_{t}=1$.

In the Appendix we show that, for a given $\mathbf{K}_{x}$, the optimum $\mathbf{K}_{w}$ in problem (7) must also be rank one, implying that beamforming is also optimal at the helper. Hence, the jamming signal can be written as $\mathbf{x}_{2}=\mathbf{w}_{h} n$ where $n$ represents a synthetic noise symbol broadcast by the helper using the beamforming vector $\mathbf{w}_{h}$. Without loss of generality, we normalize $\mathbf{w}_{h}$ to have unit length, $\mathbf{w}_{h}^{H} \mathbf{w}_{h}=1$.

Using the above facts, the optimization problem in (7) can be rewritten as

$$
\begin{aligned}
C_{\text {sec }}=\max _{\left\|\mathbf{w}_{t}\right\| \leq 1,\left\|\mathbf{w}_{h}\right\| \leq 1} \log \left(1+\frac{P_{t} \mathbf{h}_{1}^{H} \mathbf{w}_{t} \mathbf{w}_{t}^{H} \mathbf{h}_{1}}{\sigma_{r}^{2}+P_{h}\left|\mathbf{g}_{2}^{H} \mathbf{w}_{h}\right|^{2}}\right) \\
-\log \left(1+\frac{P_{t} \mathbf{g}_{1}^{H} \mathbf{w}_{t} \mathbf{w}_{t}^{H} \mathbf{g}_{1}}{\sigma_{e}^{2}+P_{h}\left|\mathbf{h}_{2}^{H} \mathbf{w}_{h}\right|^{2}}\right)
\end{aligned}
$$

From Lemma 1, it is clear that to solve the optimization problem in (10), we would need to find $\mathbf{w}_{h}$ that maximizes (8), i.e.,

$$
C_{\text {sec }}=\max _{\left\|\mathbf{w}_{h}\right\| \leq 1} C_{\text {sec }}\left(\mathbf{w}_{h}\right)=\max _{\left\|\mathbf{w}_{h}\right\| \leq 1} \log \lambda_{t}
$$

In the following we first derive an explicit expression for $\lambda_{t}$ for a given $\mathbf{w}_{h}$, and then we obtain the optimal $\mathbf{w}_{h}$ that maximizes $\lambda_{t}$.

\section{OPTIMAL BEAMFORMING AT THE HELPER AND THE TRANSMITTER}

For a given $\mathbf{w}_{h}$, we are interested in obtaining $\lambda_{t}$, which denotes the largest generalized eigenvalue of the pencil (9), and its corresponding generalized eigenvector $\mathbf{w}_{t}$. From the definition of the generalized eigenvalue decomposition we have [14]:

$$
\left(\mathbf{I}+\frac{P_{t} \mathbf{h}_{1} \mathbf{h}_{1}^{H}}{\sigma_{r}^{2}+P_{h}\left|\mathbf{g}_{2}^{H} \mathbf{w}_{h}\right|^{2}}\right) \mathbf{w}_{t}=\lambda_{t}\left(\mathbf{I}+\frac{P_{t} \mathbf{g}_{1} \mathbf{g}_{1}^{H}}{\sigma_{e}^{2}+P_{h}\left|\mathbf{h}_{2}^{H} \mathbf{w}_{h}\right|^{2}}\right) \mathbf{w}_{t} .
$$

For notational simplicity, define

$$
\begin{gathered}
\mathbf{h}=\sqrt{\frac{P_{t}}{\sigma_{r}^{2}+P_{h}\left|\mathbf{g}_{2}^{H} \mathbf{w}_{h}\right|^{2}}} \mathbf{h}_{1} \\
\mathbf{g}=\sqrt{\frac{P_{t}}{\sigma_{e}^{2}+P_{h}\left|\mathbf{h}_{2}^{H} \mathbf{w}_{h}\right|^{2}}} \mathbf{g}_{1} .
\end{gathered}
$$

Using (13) and (14) in (12), we have:

$$
\left(\mathbf{I}+\mathbf{h} \mathbf{h}^{H}\right) \mathbf{w}_{t}=\lambda_{t}\left(\mathbf{I}+\mathbf{g} \mathbf{g}^{H}\right) \mathbf{w}_{t} .
$$

It is easy to verify that among the $n_{t}$ generalized eigenvalues of the pencil $\left(\mathbf{I}+\mathbf{h h}^{H}, \mathbf{I}+\mathbf{g} \mathbf{g}^{H}\right),\left(n_{t}-2\right)$ of them are equal to 1 and are obtained using any vector that is orthogonal to the space spanned by $[\mathbf{h} \mathbf{g}]$. The two generalized eigenvectors corresponding to the maximum $\left(\lambda_{t}\right)$ and the minimum generalized eigenvalues lie completely in $\operatorname{span}([\mathbf{h} \mathbf{g}])$. Thus, we can write $\mathbf{w}_{t}$ as

$$
\mathbf{w}_{t}=\beta_{1} \mathbf{P}_{g}^{\perp} \mathbf{h}+\beta_{2} \mathbf{P}_{g} \mathbf{h}
$$

for some complex numbers $\beta_{1}$ and $\beta_{2}$ that must be solved for, where $\mathbf{P}_{g}=\left(\mathbf{g}^{H} \mathbf{g}\right)^{-1} \mathbf{g g}^{H}$ denotes the orthogonal projection onto $\operatorname{span}(\mathbf{g})$ and $\mathbf{P}_{g}^{\perp}=\mathbf{I}-\mathbf{P}_{g}$ denotes the projection onto its orthogonal complement.

From (15), we have

$$
\left(\mathbf{I}+\mathbf{g} \mathbf{g}^{H}\right)^{-1}\left(\mathbf{I}+\mathbf{h} \mathbf{h}^{H}\right) \mathbf{w}_{t}=\lambda_{t} \mathbf{w}_{t} .
$$

Note that $\left(\mathbf{I}+\mathbf{g ~ g ~}^{H}\right)^{-1}=\left(\mathbf{P}_{g}^{\perp}+\left(\mathbf{g}^{H} \mathbf{g}+1\right)^{-1} \mathbf{P}_{g}\right)$. Now it is easy to verify that

$$
\mathbf{w}_{t}=\frac{\left(-b+\sqrt{b^{2}+4 a c}\right) \mathbf{P}_{g}^{\perp} \mathbf{h}+2 a \mathbf{P}_{g} \mathbf{h}}{\left\|\left(-b+\sqrt{b^{2}+4 a c}\right) \mathbf{P}_{g}^{\perp} \mathbf{h}+2 a \mathbf{P}_{g} \mathbf{h}\right\|}
$$

and

$\lambda_{t}=\frac{\left(\mathbf{g}^{H} \mathbf{g}+1\right)^{-1}\left(1+\mathbf{h}^{H} \mathbf{P}_{g} \mathbf{h}\right)+\left(1+\mathbf{h}^{H} \mathbf{P}_{g}^{\perp} \mathbf{h}\right)+\sqrt{b^{2}+4 a c}}{2}$

where $b=\left(\mathbf{g}^{H} \mathbf{g}+1\right)^{-1}\left(1+\mathbf{h}^{H} \mathbf{P}_{g} \mathbf{h}\right)-\left(1+\mathbf{h}^{H} \mathbf{P}_{g}^{\perp} \mathbf{h}\right)$, $a=\left(\mathbf{g}^{H} \mathbf{g}+1\right)^{-1}\left(\mathbf{h}^{H} \mathbf{P}{ }_{g}^{\perp} \mathbf{h}\right)$, and $c=\left(\mathbf{h}^{H} \mathbf{P}_{g} \mathbf{h}\right)$.

It should be noted that, for any real-valued $\theta, e^{j \theta} \mathbf{w}_{t}$ is also an optimal beamforming vector at the transmitter for a given $\mathbf{w}_{h}$. Moreover, from (19) it is clear that $\lambda_{t} \geq 1$. Finally, using (13) and (14), $\mathbf{w}_{t}$ and $\lambda_{t}$ can be represented based on $\mathbf{w}_{h}$ and other channel parameters $\mathbf{h}_{1}, \mathbf{h}_{2}$, etc. To do so, it is clear that in (18) and (19) and in the definition of $a, b$ and $c$ above, $\mathbf{P}_{g}$ and $\mathbf{P}_{g}^{\perp}$ can be replaced by $\mathbf{P}_{g_{1}}$ and $\mathbf{P}_{g_{1}}^{\perp}$, respectively, where $\mathbf{P}_{g_{1}}=\left(\mathbf{g}_{1}^{H} \mathbf{g}_{1}\right)^{-1} \mathbf{g}_{1} \mathbf{g}_{1}^{H}$ and $\mathbf{P}_{g_{1}}^{\perp}=\mathbf{I}-\mathbf{P}_{g_{1}}$. Hence, for example, $c$ becomes $c=\frac{P_{t}}{\sigma_{r}^{2}+P_{h}\left|\mathbf{g}_{2}^{H} \mathbf{w}_{h}\right|^{2}}\left(\mathbf{h}_{1}^{H} \mathbf{P}_{g_{1}} \mathbf{h}_{1}\right)$.

So far, we have obtained closed-form expressions for $\lambda_{t}$ and the beamforming vector $\mathbf{w}_{t}$, for a given $\mathbf{w}_{h}$. From (11), the optimal $\mathbf{w}_{h}$ should maximize $\lambda_{t}$, with the constraint $\left\|\mathbf{w}_{h}\right\|=$ 1. The Lagrangian function $\mathcal{L}$ associated with this problem is given by

$$
\mathcal{L}=-\lambda_{t}+\zeta \mathbf{w}_{h}^{H} \mathbf{w}_{h}
$$

where $\zeta \geq 0$ is the Lagrange multiplier. By applying the KKT necessary conditions $\frac{\partial \mathcal{L}}{\partial \mathbf{w}_{h}}=0$ and $\left\|\mathbf{w}_{h}\right\|=1$ for the optimal $\mathbf{w}_{h}$, we have

$$
\begin{array}{r}
P_{h} \frac{\partial \lambda_{t}}{\partial \alpha_{1}} \mathbf{g}_{2} \mathbf{g}_{2}^{H} \mathbf{w}_{h}+P_{h} \frac{\partial \lambda_{t}}{\partial \alpha_{2}} \mathbf{h}_{2} \mathbf{h}_{2}^{H} \mathbf{w}_{h}=\zeta \mathbf{w}_{h} \\
\zeta=P_{h} \frac{\partial \lambda_{t}}{\partial \alpha_{1}} \mathbf{w}_{h}^{H} \mathbf{g}_{2} \mathbf{g}_{2}^{H} \mathbf{w}_{h}+P_{h} \frac{\partial \lambda_{t}}{\partial \alpha_{2}} \mathbf{w}_{h}^{H} \mathbf{h}_{2} \mathbf{h}_{2}^{H} \mathbf{w}_{h}
\end{array}
$$


where we define $\alpha_{1}=\sigma_{r}^{2}+P_{h} \mathbf{w}_{h}^{H} \mathbf{g}_{2} \mathbf{g}_{2}^{H} \mathbf{w}_{h}$ and $\alpha_{2}=\sigma_{e}^{2}+$ $P_{h} \mathbf{w}_{h}^{H} \mathbf{h}_{2} \mathbf{h}_{2}^{H} \mathbf{w}_{h}$. Note that (12) can be rewritten as

$$
\left(\mathbf{I}+\frac{P_{t}}{\alpha_{1}} \mathbf{h}_{1} \mathbf{h}_{1}^{H}\right) \mathbf{w}_{t}=\lambda_{t}\left(\mathbf{I}+\frac{P_{t}}{\alpha_{2}} \mathbf{g}_{1} \mathbf{g}_{1}^{H}\right) \mathbf{w}_{t} .
$$

To obtain $\frac{\partial \lambda_{t}}{\partial \alpha_{1}}$ and $\frac{\partial \lambda_{t}}{\partial \alpha_{2}}$, we use a result from [15] which states that, for two positive definite matrices $\mathbf{A}$ and $\mathbf{B}$ with generalized eigenvector $\boldsymbol{\psi}$ and eigenvalue $\mu$, so that $\mathbf{A} \boldsymbol{\psi}=\mu \mathbf{B} \psi$, and for a real parameter $\rho$ with $\mathbf{A}^{\prime}=\frac{\partial \mathbf{A}}{\partial \rho}$ and $\mathbf{B}^{\prime}=\frac{\partial \mathbf{B}}{\partial \rho}$, we have

$$
\frac{\partial \mu}{\partial \rho}=\frac{\boldsymbol{\psi}^{H}\left(\mathbf{A}^{\prime}-\mu \mathbf{B}^{\prime}\right) \boldsymbol{\psi}}{\boldsymbol{\psi}^{H} \mathbf{B} \boldsymbol{\psi}}
$$

Thus, using (23) and (24), we may write

$$
\begin{aligned}
\frac{\partial \lambda_{t}}{\partial \alpha_{1}} & =\frac{-P_{t}}{\alpha_{1}^{2}} \frac{\mathbf{w}_{t}^{H} \mathbf{h}_{1} \mathbf{h}_{1}^{H} \mathbf{w}_{t}}{\mathbf{w}_{t}^{H}\left(\mathbf{I}+\frac{P_{t}}{\alpha_{2}} \mathbf{g}_{1} \mathbf{g}_{1}^{H}\right) \mathbf{w}_{t}} \\
\frac{\partial \lambda_{t}}{\partial \alpha_{2}} & =\lambda_{t} \frac{P_{t}}{\alpha_{2}^{2}} \frac{\mathbf{w}_{t}^{H} \mathbf{g}_{1} \mathbf{g}_{1}^{H} \mathbf{w}_{t}}{\mathbf{w}_{t}^{H}\left(\mathbf{I}+\frac{P_{t}}{\alpha_{2}} \mathbf{g}_{1} \mathbf{g}_{1}^{H}\right) \mathbf{w}_{t}}
\end{aligned}
$$

Now, by replacing Eqs. (22), (25) and (26) in (21), we have

$$
\begin{gathered}
\left(\lambda_{t} \frac{\left|\mathbf{g}_{1}^{H} \mathbf{w}_{t}\right|^{2}}{\alpha_{2}^{2}} \mathbf{h}_{2} \mathbf{h}_{2}^{H}-\frac{\left|\mathbf{h}_{1}^{H} \mathbf{w}_{t}\right|^{2}}{\alpha_{1}^{2}} \mathbf{g}_{2} \mathbf{g}_{2}^{H}\right) \mathbf{w}_{h}= \\
\left(\lambda_{t} \frac{\left|\mathbf{g}_{1}^{H} \mathbf{w}_{t}\right|^{2}}{\alpha_{2}^{2}}\left|\mathbf{h}_{2}^{H} \mathbf{w}_{h}\right|^{2}-\frac{\left|\mathbf{h}_{1}^{H} \mathbf{w}_{t}\right|^{2}}{\alpha_{1}^{2}}\left|\mathbf{g}_{2}^{H} \mathbf{w}_{h}\right|^{2}\right) \mathbf{w}_{h}
\end{gathered}
$$

Eq. (27) shows that the optimal $\mathbf{w}_{h}$ is in fact an eigenvector of the matrix $\lambda_{t} \frac{\left|\mathbf{g}_{1}^{H} \mathbf{w}_{t}\right|^{2}}{\alpha_{2}^{2}} \mathbf{h}_{2} \mathbf{h}_{2}^{H}-\frac{\left|\mathbf{h}_{1}^{H} \mathbf{w}_{t}\right|^{2}}{\alpha_{1}^{2}} \mathbf{g}_{2} \mathbf{g}_{2}^{H}$. Since, in Eq. (27), $\alpha_{1}, \alpha_{2}, \mathbf{w}_{t}$ and $\lambda_{t}$ are functions of $\mathbf{w}_{h}$, finding a closed-form expression for the optimal $\mathbf{w}_{h}$ is intractable. Instead, it is easy to show that $\mathbf{w}_{h}$ must have the following form [11]

$$
\mathbf{w}_{h}=\cos (\theta) \frac{\mathbf{P}_{g_{2}}^{\perp} \mathbf{h}_{2}}{\left|\mathbf{P}_{g_{2}}^{\perp} \mathbf{h}_{2}\right|}+\sin (\theta) \frac{\mathbf{P}_{g_{2}} \mathbf{h}_{2}}{\left|\mathbf{P}_{g_{2}} \mathbf{h}_{2}\right|} \quad 0 \leq \theta \leq \pi / 2
$$

where $\mathbf{P}_{g_{2}}=\left(\mathbf{g}_{2}^{H} \mathbf{g}_{2}\right)^{-1} \mathbf{g}_{2} \mathbf{g}_{2}^{H}$, and $\mathbf{P}_{g_{2}}^{\perp}=\mathbf{I}-\mathbf{P}_{g_{2}}$. Note that zero-forcing (ZF) as a sub-optimal solution is obtained for $\theta=0$. But the optimal $\mathbf{w}_{h}$ is obtained by searching over $0 \leq \theta \leq \pi / 2$ and finding the $\theta$ for which (27) is satisfied.

Finally, the secrecy capacity of this channel is given by

$$
C_{s e c}=\log \lambda_{t}^{*}
$$

where $\lambda_{t}^{*}$ is given by (19) for $\mathbf{w}_{h}^{*}$ in (27). The optimal beamforming vector at the transmitter $\mathrm{w}_{t}^{*}$ that achieves (29) is given by (18) for $\mathbf{w}_{h}^{*}$ in (27). This clarifies the difference between our work and [11], where an iterative algorithm is required to jointly optimize the transmitter and helper beamformers.

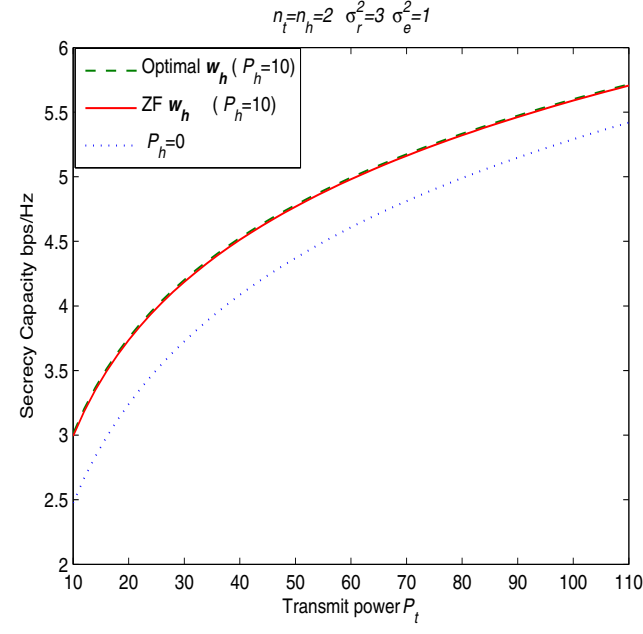

Fig. 1. Comparison of secrecy capacity for MISO Gaussian wiretap channel with and without helper versus $P_{t}$, for uncorrelated channels.

\section{NUMERICAL RESULTS}

In this section, we present numerical results to illustrate our theoretical findings. The channel vectors $\mathbf{h}_{1} \in \mathbb{C}^{n_{t} \times 1}$ and $\mathbf{h}_{2} \in \mathbb{C}^{n_{h} \times 1}$ have entries distributed as $\mathcal{C N}\left(0, \sigma_{d}^{2}\right)$, while $\mathbf{g}_{1} \in \mathbb{C}^{n_{t} \times 1}$ and $\mathbf{g}_{2} \in \mathbb{C}^{n_{h} \times 1}$ have entries distributed as $\mathcal{C N}\left(0, \sigma_{c}^{2}\right)$. Results are calculated based on an average of at least 10000 independent channel realizations.

Fig. 1 shows the secrecy capacity versus $P_{t}$ when $n_{t}=$ $n_{h}=2, \sigma_{d}^{2}=2, \sigma_{c}^{2}=1, \sigma_{r}^{2}=3$, and $\sigma_{e}^{2}=1$. In this case, the channel vectors are all uncorrelated. We compare the secrecy capacity without a helper $\left(P_{h}=0\right)$ to what can be obtained with a helper assuming $P_{h}=10$. Results are plotted for both the optimal $\mathbf{w}_{h}$ as well as the sub-optimal zero-forcing solution obtained for $\theta=0$ in (28). The figure shows that even a helper with low power provides a considerable benefit, and the benefit is maintained even if the total transmit power $P_{h}+P_{t}$ is held constant. The figure also shows that the secrecy rate of the ZF strategy is very close to the secrecy capacity. Fig. 2 shows the results for the case where the channel pairs $\left\{\mathbf{g}_{1}, \mathbf{h}_{1}\right\}$ and $\left\{\mathbf{g}_{2}, \mathbf{h}_{2}\right\}$ are highly correlated (correlation coefficient of 0.95 ). While the optimal $\mathbf{w}_{h}$ provides a benefit over the ZF strategy, it is very small and the secrecy capacity is nearly zero anyway.

\section{CONCLUSIONS}

In this paper, we have studied the Gaussian MISO wiretap channel in the presence of an external jammer/helper, where the helper node assists the transmitter by sending artificial noise independent of the source message to confuse the eavesdropper. The jamming signal from the helper is not decoded by the intended receiver and is treated as noise at both the in- 


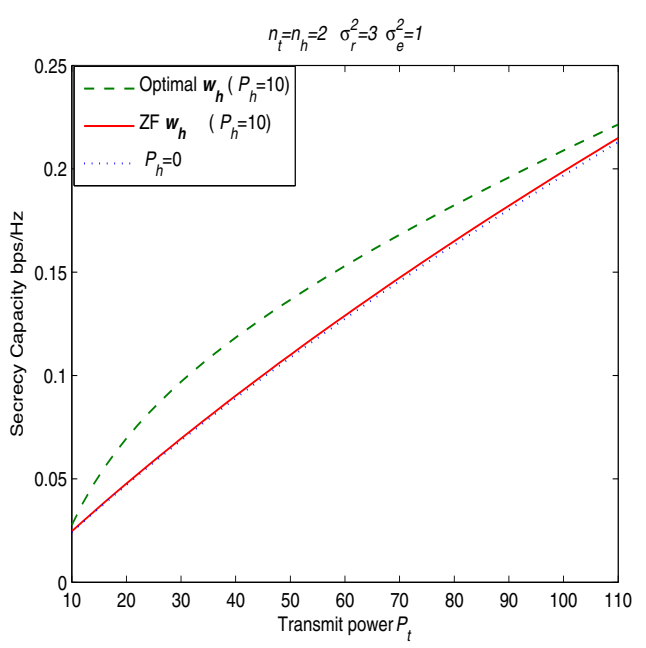

Fig. 2. Comparison of secrecy capacity for MISO Gaussian wiretap channel with and without helper versus $P_{t}$, for correlated channels.

tended receiver and the eavesdropper. We showed how to obtain optimal transmit beamformers at the transmitter and the helper, but we demonstrated that using a zero-forcing beamformer at the helper is a near-optimal choice for obtaining the secrecy capacity in this scenario.

\section{APPENDIX}

For a given $\mathbf{K}_{x}$, the Lagrangian function $\mathcal{L}$ associated with problem (7) is given by

$$
\begin{array}{r}
\mathcal{L}=\log \left(1+\frac{\mathbf{h}_{1}^{H} \mathbf{K}_{x} \mathbf{h}_{1}}{\sigma_{r}^{2}+\mathbf{g}_{2}^{H} \mathbf{K}_{w} \mathbf{g}_{2}}\right)-\log \left(1+\frac{\mathbf{g}_{1}^{H} \mathbf{K}_{x} \mathbf{g}_{1}}{\sigma_{e}^{2}+\mathbf{h}_{2}^{H} \mathbf{K}_{w} \mathbf{h}_{2}}\right) \\
-\zeta \operatorname{Tr}\left(\mathbf{K}_{w}\right)+\operatorname{Tr}\left(\mathbf{M} \mathbf{K}_{w}\right)
\end{array}
$$

where $\zeta>0$ is the Lagrange multiplier and $\mathbf{M} \succeq \mathbf{0}$. Let $\mathbf{K}_{w}^{*}$ be an optimal solution of problem (30). Then $\mathbf{K}_{w}^{*}$ must satisfy the following Karush-Kuhn-Tucker (KKT) conditions:

$$
\begin{aligned}
\mathbf{M} & =\zeta \mathbf{I}+\delta_{g} \mathbf{g}_{2} \mathbf{g}_{2}^{H}-\delta_{h} \mathbf{h}_{2} \mathbf{h}_{2}^{H} \\
\mathbf{K}_{w}^{*} \mathbf{M} & =\mathbf{0}
\end{aligned}
$$

where $\delta_{g}=\left(\sigma_{r}^{2}+\mathbf{g}_{2}^{H} \mathbf{K}_{w}^{*} \mathbf{g}_{2}\right)^{-1}-\left(\mathbf{h}_{1}^{H} \mathbf{K}_{x} \mathbf{h}_{1}+\sigma_{r}^{2}+\mathbf{g}_{2}^{H} \mathbf{K}_{w}^{*} \mathbf{g}_{2}\right)^{-1}$ and $\delta_{h}=\left(\sigma_{e}^{2}+\mathbf{h}_{2}^{H} \mathbf{K}_{w}^{*} \mathbf{h}_{2}\right)^{-1}-\left(\mathbf{g}_{1}^{H} \mathbf{K}_{x} \mathbf{g}_{1}+\sigma_{e}^{2}+\mathbf{h}_{2}^{H} \mathbf{K}_{w}^{*} \mathbf{h}_{2}\right)^{-1}$ are positive real parameters. From (32), in order to show that $\operatorname{rank}\left(\mathbf{K}_{w}^{*}\right)=1$, it suffices to show that $\operatorname{rank}(\mathbf{M})=n_{h}-1$. Define $\mathbf{B}=\zeta \mathbf{I}+\delta_{g} \mathbf{g}_{2} \mathbf{g}_{2}^{H}$. Using a technique similar to that proposed in [16], we have

$$
\begin{aligned}
\operatorname{rank}(\mathbf{M}) & =\operatorname{rank}\left(\mathbf{B}^{-1 / 2}\left(\mathbf{B}-\delta_{h} \mathbf{h}_{2} \mathbf{h}_{2}^{H}\right) \mathbf{B}^{-1 / 2}\right) \\
& =\operatorname{rank}\left(\mathbf{I}-\delta_{h}\left(\mathbf{B}^{-1 / 2} \mathbf{h}_{2}\right)\left(\mathbf{B}^{-1 / 2} \mathbf{h}_{2}\right)^{H}\right)=n_{h}-1 .
\end{aligned}
$$

\section{REFERENCES}

[1] A. Wyner, "The wire-tap channel," Bell. Syst. Tech. J., vol. 54, no. 8, pp. 1355-1387, Jan. 1975.

[2] A. Khisti and G. Wornell, "Secure transmission with multiple antennas I: The MISOME wiretap channel," IEEE Trans. Inf. Theory, vol. 56, no. 7, pp. 3088-3104, 2010.

[3] S. Ali. A. Fakoorian and A. L. Swindlehurst, "Optimal power allocation for the GSVD based MIMO Gaussian wiretap channel," submitted to IEEE Trans. Inf. Theory, Available: http://arxiv.org/abs/1006.1890

[4] T. Liu and S. Shamai (Shitz), "A note on secrecy capacity of the multi-antenna wiretap channel," IEEE Trans. Inf. Theory, vol. 55, no. 6, pp. 2547-2553, 2009.

[5] R. Bustin, R. Liu, H. V. Poor, and S. Shamai (Shitz), "A MMSE approach to the secrecy capacity of the MIMO Gaussian wiretap channel," EURASIP Journal on Wireless Communications and Networking, vol. 2009.

[6] I. Csiszar and J. Korner, "Broadcast channels with confidential messages," IEEE Trans. Inf. Theory, vol. 24, pp. 339-348, May 1978.

[7] E. Tekin and A. Yener, "The general Gaussian multiple access and two-way wire-tap channels: Achievable rates and cooperative jamming," IEEE Trans. Inf. Theory, vol. 54, no. 6, pp. 27352751, Jun. 2008.

[8] L. Dong, Z. Han, A. P. Petropulu, and H. V. Poor, "Improving wireless physical layer security via cooperating relays," IEEE Trans. Signal Proc., vol. 58, NO. 3, pp. 1875-1888, Mar. 2010.

[9] L. Lai and H. El Gamal, "The relay-eavesdropper channel: Cooperation for secrecy," IEEE Trans. Inf. Theory, vol. 54, no. 9, pp. 40054019, Sep. 2008.

[10] X. Tang, R. Liu, P. Spasojevic, and H. V. Poor, "The Gaussian wiretap channel with a helping interferer," in Proc. ISIT 2008, Toronto, ON, Canada, Jul. 2008.

[11] E. A. Jorswieck, "Secrecy capacity of single- and multiantenna channels with simple helpers," in Proc. IEEE Int. Conf. Source and Channel Coding (SCC), April 2010.

[12] J. Wang and A. Swindlehurst, "Cooperative jamming in MIMO ad hoc networks," in Proc. Asilomar Conf. on Signals, Systems and Computers, Nov., 2009.

[13] R. Liu, and H. Vincent Poor, "Secrecy capacity region of a multiple-antenna Gaussian broadcast channel with confidential messages," IEEE Trans. Inf. Theory, vol. 55 no. 3, March. 2009.

[14] R. A. Horn and C. R. Johnson, Matrix Analysis, University Press, Cambridge, UK, 1985.

[15] R. L. Dailey, "Eigenvector derivatives with repeated eigenvalues," AIAA JOURNAL, vol. 27, no. 4, April 1989.

[16] W. C. Liao, T. H. Chang, W. K Ma, and C. Y. Chi, "Joint transmit beamforming and artificial noise design for QoS discrimination inwireless downlink," ICASSP 2010, pp. 2562-2565, March 2010. 